\title{
Using IQ Discrepancy Scores To Examine the Neural Correlates of Specific Cognitive Abilities
}

\author{
Amy Margolis, ${ }^{1,2}$ Ravi Bansal, ${ }^{1,2}$ Xuejun Hao, ${ }^{1,2}$ Molly Algermissen, ${ }^{1}$ Cole Erickson, ${ }^{1,2}$ Kristin W. Klahr, ${ }^{1,2}$ \\ Jack A. Naglieri, ${ }^{3}$ and Bradley S. Peterson ${ }^{1,2}$ \\ ${ }^{1}$ Center for Developmental Neuropsychiatry, Department of Psychiatry, Columbia University College of Physicians and Surgeons, New York, New York \\ 10032, ${ }^{2}$ New York State Psychiatric Institute, New York, New York 10032, and 3'University of Virginia, Charlottesville, Virginia 22904
}

The underlying neural determinants of general intelligence have been studied intensively, and seem to derive from the anatomical and functional characteristics of a frontoparietal network. Little is known, however, about the underlying neural correlates of domain-specific cognitive abilities, the other factors hypothesized to explain individual performance on intelligence tests. Previous preliminary studies have suggested that spatially distinct neural structures do not support domain-specific cognitive abilities. To test whether differences between abilities that affect performance on verbal and performance tasks derive instead from the morphological features of a single anatomical network, we assessed in two independent samples of healthy human participants $(N=83$ and $N=58$; age range, 5-57 years) the correlation of cortical thickness with the magnitude of the verbal intelligence quotient (VIQ)-performance intelligence quotient (PIQ) discrepancy. We operationalized the VIQ-PIQ discrepancy by regressing VIQ onto PIQ (VIQ-regressed-on-PIQ score), and by regressing PIQ onto VIQ (PIQ-regressed-on-VIQ score). In both samples, a progressively thinner cortical mantle in anterior and posterior regions bilaterally was associated with progressively greater (more positive) VIQ-regressed-on-PIQ scores. A progressively thicker cortical mantle in anterior and posterior regions bilaterally was associated with progressively greater (more positive) PIQ-regressed-on-VIQ scores. Variation in cortical thickness in these regions accounted for a large portion of the overall variance in magnitude of the VIQ-PIQ discrepancy. The degree of hemispheric asymmetry in cortical thickness accounted for a much smaller but statistically significant portion of variance in VIQ-PIQ discrepancy.

\section{Introduction}

For over a century, psychologists have been interested in measuring individual intelligence. Modern intelligence quotient (IQ) tests are based either on a general model of intelligence (Wechsler, 2008) or on a multidimensional model of intelligence (Naglieri and Das, 1997). Neuroimaging studies have begun to investigate the neural determinants of intelligence. Most have focused on identifying the neural correlates of general intelligence $(g)$ using the Wechsler scales of intelligence. The construct of general intelligence is validated by the positive intercorrelation of subtest scores in IQ tests, sometimes referred to as the "positive manifold" (Carroll, 1993; Colom and Thompson, 2011), and is thought to derive from the overall connectivity of multimodal association regions in the brain (Shaw et al., 2006; Jung and Haier, 2007; Narr et al., 2007; Karama et al., 2009). Lesion studies, however, demonstrate dissociable cognitive skills that are better explained by multidimensional models of intelligence. The neu-

\footnotetext{
Received Feb. 19, 2013; revised June 21, 2013; accepted July 16, 2013.

Author contributions: A.M. and B.S.P. designed research; A.M. and K.W.K. performed research; A.M., R.B., X.H., C.E., and B.S.P. analyzed data; A.M., M.A., C.E., J.A.N., and B.S.P. wrote the paper.

This research was supported by National Institute of Mental Health Grants 2T32 MH16434, MH089582, K02

74677, MH068318, and MH59139 to B.S.P.; and by the Laura Lemle Family Foundation and The NVLD Project.

The authors declare no competing financial interests.

Correspondence should be addressed to Bradley S. Peterson, NYSPI, 1051 Riverside Drive, Unit 74, New York, NY 10032.E-mail: petersob@nyspi.columbia.edu.

DOI:10.1523/JNEUROSCI.0775-13.2013

Copyright $\odot 2013$ the authors $\quad 0270-6474 / 13 / 3314135-11 \$ 15.00 / 0$
}

ral determinants of these multiple dimensions, however, remain unknown.

Models of intelligence derive in large part from factor analyses, a mathematical technique that reduces correlational data to a smaller number of unobservable latent factors. The intercorrelation of performance on IQ subtests, shared variance, accounts for $50 \%$ of the total variance in performance. Roughly $70 \%$ of this shared variance represents $g$. The remaining $30 \%$ of variance is captured by factors representing domain-specific abilities (DSAs; Watkins, 2006). These DSAs vary with the differing IQ tests included in a factor analysis (Flanagan et al., 2000).

The few studies examining the neural correlates of DSAs have produced equivocal results (Andreasen et al., 1993; Gray et al., 2003; Witelson et al., 2006; Luders et al., 2007; Lange et al., 2010). In one study, g-adjusted verbal IQ (VIQ) and performance IQ (PIQ) scores did not correlate with cortical thickness (CT; Karama et al., 2011), suggesting that DSAs do not derive from spatially discrete, independent neural systems. The current study was designed to examine the neural systems that support intraindividual discrepancies in verbal- and performance-specific abilities. Following the methods used in recent studies (Karama et al., 2011), we regressed VIQ onto PIQ (VIQ-regressed-on-PIQ score) and PIQ onto VIQ (PIQ-regressed-on-VIQ score) and correlated the residual scores with $\mathrm{CT}$. The shared variance between VIQ and PIQ represents variance associated with $g$, and the residual represents variance associated with domain-specific cognitive abilities and task-specific skills. The residual scores that we 
calculated index intraindividual variability in performance across VIQ- and PIQ-associated tasks. No other study, to our knowledge, has identified the neural correlates of the VIQ-PIQ discrepancy.

The VIQ-PIQ discrepancy may correlate with a single network of brain regions or with multiple networks. As prior studies did not identify discrete neural correlates of $g$-adjusted VIQ and PIQ, we did not expect the VIQ-PIQ discrepancy to depend on variation in multiple networks. We hypothesized instead that a single neural system underlies the imbalance between verbal and perceptual scores, and therefore that the VIQ-regressed-on-PIQ and PIQ-regressed-on-VIQ scores would correlate with CT in a single network of brain regions. A discrete network could explain discrepancies among DSAs by mediating competition between them, similar to explanations for the competition observed between memory systems in animals and humans (Packard, 2009). Moreover, detecting significant correlations in regions other than those associated with $g$ would suggest that a neural system distinct from $g$ governs the VIQ-PIQ discrepancy.

\section{Materials and Methods}

The Institutional Review Board at New York State Psychiatric Institute approved the studies from which these data are drawn. All parents gave consent for children to participate. Children gave their assent to participate. All participants were paid for their participation.

\section{Participants}

Two independent samples of healthy control individuals were included in this study. For both, we recruited healthy adults and children selected randomly from a telemarketing database. Recruitment methods for these studies have been reported previously (Goh et al., 2011). All participants were recruited as controls for imaging studies of adult and childhood neuropsychiatric disorders. Introductory letters were sent and then followed by screening telephone calls. A detailed clinical interview was performed that included the Kiddie-Schedule for Affective Disorders and Schizophrenia for children (Kaufman et al., 1997) and the Structured Clinical Interview for the Diagnostic and Statistical Manual of Mental Disorders, fourth edition, Axis I Disorders (Spitzer et al., 1992) for adults. Two independent clinicians reviewed each file and rated each participant. Individuals with a current or past history of a psychiatric or neurological disorder were excluded.

Sample 1. This sample included 83 participants with a Full Scale IQ (FSIQ) of $\geq 80$, who ranged in age from 5 to 54 years (mean age, 19.38 years; SD, 11.98 years). The sample was similarly distributed across gender (females, 40\%) and was predominantly right handed (94\%). Children under 18 years of age comprised $60 \%$ of the sample (Table 1).

Sample 2. This sample included 58 participants with an FSIQ of $\geq 80$, who ranged in age from 7 to 57 years (mean age, 21.70 years; SD, 13.21 years). The sample was similarly distributed across gender (females, $50 \%)$ and was right handed $(100 \%)$. Children under 18 years of age comprised $45 \%$ of the sample (Table 1 ).

\section{Neuropsychological assessment}

Because healthy participants were recruited as controls for several different studies across two university settings, intelligence testing was performed using different instruments depending on the research study protocol. Intelligence testing was performed in close temporal proximity to magnetic resonance imaging (MRI) scanning.

Sample 1. The majority of participants completed the Wechsler Abbreviated Scale of Intelligence (WASI; Wechsler, 1999) using the foursubtest format $(n=67)$. Sixteen children completed the Wechsler Intelligence Scale for Children, third edition (WISC-III; Wechsler, 1991). FSIQ correlations between the WASI and longer forms have been established at $0.87-0.92$.

Sample 2. Fifteen children completed the WISC-III; abbreviated IQ indexes were estimated using established methods that correlate scores of $\geq 0.90$ with scores from the full administration (Kaufman et al., 1996).
Table 1. Demographic data for all subjects separated by sample

\begin{tabular}{|c|c|c|c|c|c|c|c|c|}
\hline & \multicolumn{4}{|c|}{ Sample 1} & \multicolumn{4}{|c|}{ Sample 2} \\
\hline & \multicolumn{2}{|c|}{ Children } & \multicolumn{2}{|c|}{ Adults $^{a}$} & \multicolumn{2}{|c|}{ Children } & \multicolumn{2}{|c|}{ Adults $^{a}$} \\
\hline & Male & Female & Male & Female & Male & Female & Male & Female \\
\hline Subjects (№.) & 27 & 23 & 23 & 10 & 12 & 14 & 17 & 15 \\
\hline Subjects (\%) & 32.5 & 27.7 & 27.7 & 12.1 & 20.6 & 24.1 & 29.2 & 25.9 \\
\hline Average age (years) & 11.6 & 11.5 & 33.8 & 25.5 & 10.9 & 9.8 & 29.2 & 32.9 \\
\hline Youngest (years) & 5.8 & 5.9 & 18.4 & 18.9 & 8.2 & 7.3 & 18.3 & 21.2 \\
\hline Oldest (years) & 17.9 & 17.5 & 54.4 & 37.77 & 15.1 & 15.6 & 53.7 & 57.2 \\
\hline Min VIQ & 75 & 73 & 92 & 95 & 91 & 85 & 110 & 91 \\
\hline Max VIQ & 139 & 149 & 141 & 150 & 135 & 129 & 150 & 150 \\
\hline Average VIQ & 107.5 & 109.2 & 121.1 & 115.4 & 112.3 & 108.3 & 133.8 & 120.7 \\
\hline Min PIQ & 86 & 85 & 96 & 93 & 104 & 86 & 92 & 104 \\
\hline Max PIQ & 135 & 144 & 137 & 128 & 150 & 140 & 150 & 146 \\
\hline Average PIQ & 108.7 & 112.8 & 119 & 105 & 121.6 & 113.4 & 118.8 & 121.6 \\
\hline Min FSIQ & 80 & 86 & 86 & 100 & 103 & 90 & 107 & 96 \\
\hline Max FSIQ & 134 & 142 & 137 & 144 & 143 & 131 & 150 & 150 \\
\hline Average FSIQ & 108.7 & 112 & 119 & 113 & 118.8 & 111.9 & 130.1 & 123.7 \\
\hline
\end{tabular}

Min, Minimum; Max, maximum.

${ }^{a}$ Over 18 years of age.

The WISC-III four-subtest administration included Vocabulary and Information subtests to estimate VIQ, Block Design and Object Assembly to estimate PIQ, and the sum of these four tests to estimate Full Scale IQ. Thirty adults completed the Wechsler Adult Intelligence Scale (WAIS)Revised (Wechsler, 1981). Three children and two adults completed the WASI. Eight children completed the Kaufmann Brief Test of Intelligence (K-BIT; Kaufman and Kaufman, 1985). The K-BIT included Vocabulary for VIQ and Matrices for PIQ. Correlations of the K-BIT with WISC-III have been established at 0.89 (Canivez et al., 2005).

Although different instruments were used to calculate VIQ, PIQ, and FSIQ (in part, because of the wide age range of participants and, in part, because of differing study protocols), all instruments have been shown to correlate highly with one another and to test similar domains of cognitive function. Performance measures of the WASI (Block Design and Matrix Reasoning) correlate 0.60 and 0.54, respectively, with Perceptual Organization in the WISC-III, and 0.73 and 0.68 , respectively, with the WAIS, third edition (WAIS-III; Kamphaus, 2005). Verbal measures of the WASI (Vocabulary and Similarities) are included in the WISC-III and WAIS-III, and correlate 0.87 and 0.66 , respectively, with the WISC-III and 0.90 and 0.75 with the WAIS-III Verbal Comprehension factor (Kamphaus, 2005). We note that if all participants had been administered the same IQ test, measurement variance would have been reduced and our findings would likely have been stronger. That the findings were so strong and reproducible across datasets, despite the inclusion of some participants who were administered different IQ tests, is a testament to the robustness of our findings.

\section{Residual scores}

To create the VIQ-regressed-on-PIQ score, we regressed VIQ onto PIQ with an intercept of zero and saved the residual. To create the PIQregressed-on-VIQ score, we regressed PIQ onto VIQ with an intercept of zero and saved the residual.

\section{Standardized difference scores}

We assessed the correlation of CT with the VIQ-PIQ discrepancy score because discrepancy scores, as opposed to residual scores, are used frequently in clinical practice. Subtracting two standardized scores creates a difference score; the difference score will have an SE of difference (SEDiff) that is different from, and smaller than, the SD of the instrument. The SEDiff score is the SD of the difference score. SEDiff depends on the reliability of the scores being compared, in this case the IQ indices VIQ and PIQ. VIQ and PIQ have different reliabilities for each IQ instrument included in our study. Only a standardized discrepancy score makes discrepancy scores comparable across instruments. This standardized discrepancy score ( $z$-score) is created using the unique 
SEDiff for each instrument (Anastasi and Urbina, 1997). The reliability of the VIQ and PIQ index scores in the WASI varies with age, and therefore the SEDiff was calculated for each group, under and over 18 years of age. For these reasons, we calculated standardized scores separately for each group of participants who completed a particular instrument and then correlated these standardized difference scores (VIQ-PIQ) $z$ with cortical thickness.

\section{IQ score distributions}

The mean VIQ scores (112.7 in sample 1; 119.8 in sample 2) and PIQ scores (112.2 in sample $1 ; 118.8$ in sample 2) in both samples were higher than in the standardization sample, wherein the mean was 100 . Nevertheless, the mean VIQ-regressed-on-PIQ and PIQ-regressed-on-VIQ scores were essentially zero, and the distribution of the range of scores was close to the normal distribution. In samples 1 and 2, respectively, the correlations of VIQ with PIQ were 0.461 and 0.309 ; the correlations of (VIQ-PIQ) z with VIQ were 0.683 and 0.635 ; the correlations of (VIQ-PIQ) $z$ with PIQ were -0.334 and -0.539 ; the correlations of VIQ-regressed-on-PIQ with VIQ were 0.684 and 0.639; the correlations of VIQ-regressed-on-PIQ with PIQ were -0.333 and -0.535 ; the correlations of PIQ-regressed-on-VIQ with VIQ were -0.672 and -0.626; and the correlations of PIQ-regressed-on-VIQ with PIQ were 0.347 and 0.549 .

\section{Image acquisition}

Sample 1. Anatomical images were acquired on a 3 tesla whole-body scanner (Signa, General Electric) with a body transmitter coil and an eight-channel head receiver coil. High-resolution, T1-weighted brain images were acquired using a fast spoiled gradient recall sequence [inversion time, $500 \mathrm{~ms}$; repetition time (TR), $4.7 \mathrm{~ms}$; echo time (TE), $1.3 \mathrm{~ms}$; field of view (FOV), $24 \mathrm{~cm}$; image matrix, $256 \times 256$; acceleration factor, 2; 160 slices; slice thickness, $1 \mathrm{~mm}$ ] encoded for sagittal slice reconstruction, providing voxel dimensions of $0.9375 \times 0.9375 \times 1.0 \mathrm{~mm}$.

Sample 2. Anatomical images were acquired using a 1.5 tesla scanner (Signa; General Electric) for each participant. Head positioning was standardized using cantho-meatal landmarks. Images were acquired using a three-dimensional spoiled gradient recall sequence with the following parameters: TR, $24 \mathrm{~ms}$; TE, $5 \mathrm{~ms}$; $45^{\circ}$ flip angle; matrix size $256 \times 192$; two excitations; FOV, $30 \mathrm{~cm}$; 124 contiguous sagittal slices that were 1.2 $\mathrm{mm}$ thick without skip and reformatted with voxel dimensions $1.17 \times$ $1.17 \times 1.2 \mathrm{~mm}$.

\section{Image processing}

Images were processed on Sun Ultra 10 workstations using ANALYZE 8.0 Biomedical Imaging Resource (Mayo Foundation, Rochester, MN) and software developed in-house; technicians were blind to subject characteristics and hemisphere (images were randomly flipped in the transverse plane before preprocessing). We resliced the MRI dataset to correct for any residual head rotation, tilt, or flexion/extension before performing morphometric analyses.

We removed inhomogeneities in image intensity before isolating cerebral tissue (Sled et al., 1998). Inter-rater reliabilities, calculated as intraclass correlation coefficients using a two-way random-effects model, were all >0.98 (Arndt et al., 1991).

Preprocessing. We corrected large-scale variations in image intensity using an algorithm developed at the Montreal Neurological Institute (Sled et al., 1998). Extracerebral tissues were removed using an automated tool for brain extraction (Shattuck and Leahy, 2002) that smoothes image intensity with an anisotropic filter, detects threedimensional (3-D) edges using a Marr-Hildreth edge detector (Marr and Hildreth, 1980), and selects as the brain the largest connected component with a closed boundary. We manually removed connecting dura on each slice in sagittal view and checked it in orthogonal views. The brainstem was transected at the pontomedullary junction.

Cortical gray matter segmentation. We sampled bilaterally gray-scale values of "pure" representations of cortical gray matter and white matter at four standard locations (frontal, temporal, occipital, and parietal lobes) using an $8 \times 8=64$ pixel array, which is sufficiently large to provide statistical stability but small enough to avoid including other tissue types, which can create partial volume effects. We averaged these four values for each tissue type, and computed a threshold value halfway between the mean gray and white matter values for a slice in the imaging volume. We used these threshold values on a slice-by-slice basis to provide an initial rough classification of cortical gray and white matter throughout the cerebrum. To provide the most accurate segmentation possible of the cortical mantle, we hand edited the classification in the coronal and transverse views. The intraclass correlation coefficient measuring the reliability of cortical gray matter volumes using a two-way random-effects model (Shrout and Fleiss, 1979) was 0.98.

Choice of template brain. To select a template brain that was most representative of our cohort, we identified a brain that was as representative as possible of the demographics of the sample (i.e., demographic data were closest to the mean as possible in terms of age, weight, and height). We then coregistered the brains of the remaining participants to this preliminary template. We determined point correspondences on the surfaces of their cortices and computed the distances of the corresponding points on the cerebral surface of the other participants from the surface of the template brain. The brain for which all points across the surface were closest (in terms of least squares) to the average of the computed distances was selected as the final template brain. We then performed a second coregistration of all brains to this most representative template. A single representative brain serves as a better template than does an averaged brain because a single brain has well defined tissue interfaces. Averaging images for a template blurs these boundaries and increases registration errors, which are important when distinguishing subtle effects across populations.

Sulcal overlay. We overlaid onto the statistical maps of our template brain the sulcal boundaries previously identified on the International Consortium for Brain Mapping (ICBM) high-resolution, singleparticipant template (http://www.loni.ucla.edu/ICBM/Downloads/ Downloads_ICBMtemplate.shtml; Mazziotta et al., 2001) to aid visual identification of the locations of findings on the surface of the brain. We mapped the sulcal boundaries and 3-D labels of cortical gyri from the ICBM template onto the cortical surface of our template brain using our high-dimensional, nonrigid warping algorithm (see above).

\section{Cortical thickness}

For detailed descriptions of our procedures for surface morphologies, and related validation studies, see previous studies (Bansal et al., 2005). In short, random flips were reversed to provide their original correct orientation. Isolated brains were coregistered to the selected template brain (Plessen et al., 2006) using a similarity transformation, followed by a high-dimensional, nonrigid warping algorithm based on the dynamics of fluid flow. We removed the cortical mantle from each coregistered brain. We then used a three-dimensional morphological operator to distance transform this brain without the cortex from the coregistered brain of the same participant that contained the cortex (Rosenfeld and Pfaltz, 1968; Haralick and Shapiro, 1991). Thus, we calculated CT as the smallest distance of each point on the external cortical surface from the outermost surface of the white matter in the coregistered brain. Because the brain and its local features, such as CT, were scaled during the similarity transformation of that brain to the template brain, these thickness values, measured in template space, inherently accounted for generalized scaling effects within the cerebrum.

\section{Cortical asymmetry}

To compare CTs across the right hemisphere with those across the left hemisphere, the right hemisphere of each brain was normalized to the template left hemisphere using a two-step process. First, the right hemisphere of each brain was flipped to create a mirror image so that it overlay the left hemisphere of the same brain. The right hemisphere was then warped to the left hemisphere by a high-dimensional, nonrigid warping algorithm based on the dynamics of fluid flow. Second, we used the deformation field that mapped the left hemisphere on the template left hemisphere to warp the right hemisphere to the template left hemisphere. This two-step process established correspondences across all points on the right hemisphere and those across the left hemisphere of the template brain, thereby allowing us to compute variations in CT at particular voxels in each hemisphere. We then assessed the correlation between the VIQ-regressed-on-PIQ score and the left-right CT differ- 


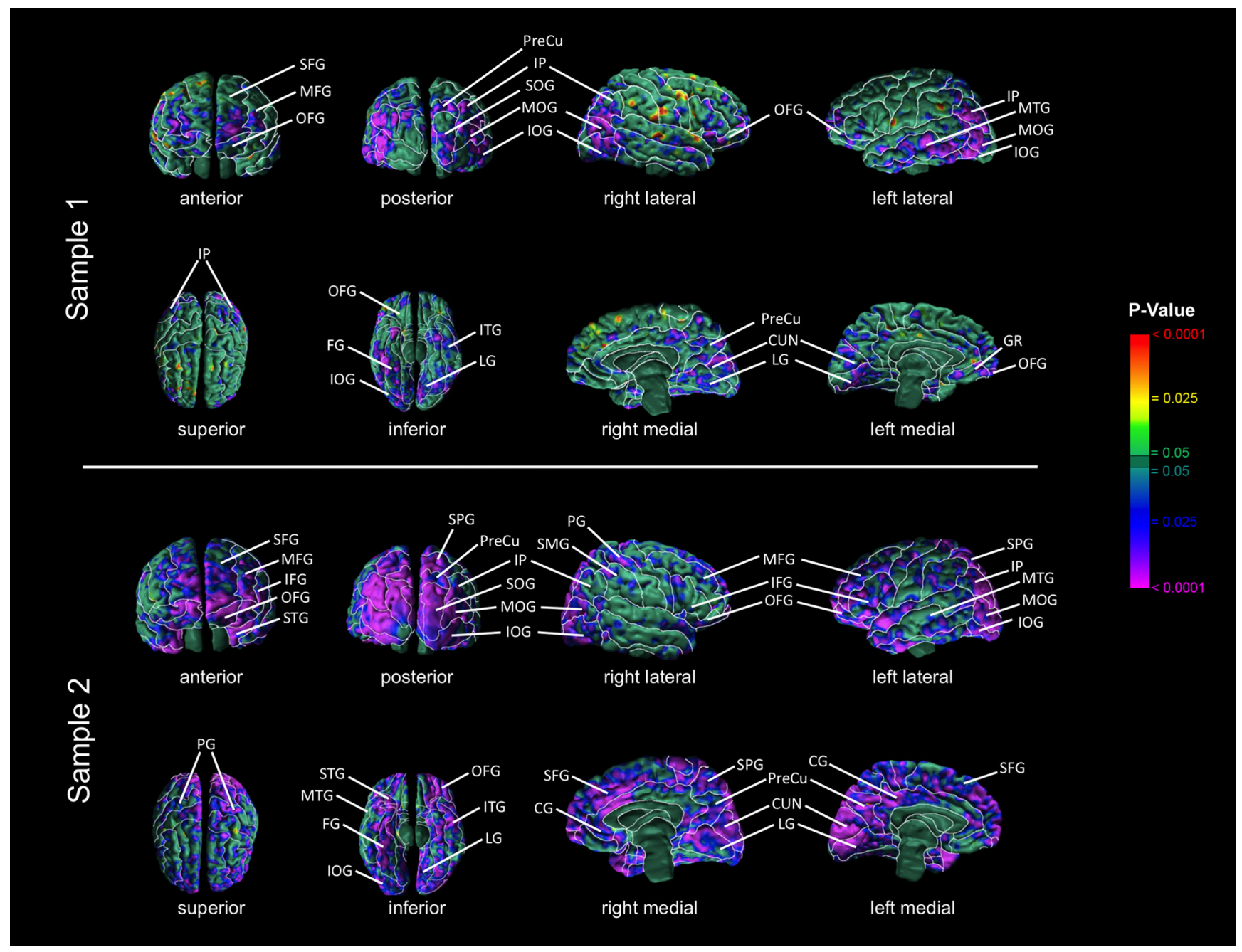

Figure 1. Significance maps showing correlations of cortical thickness (CT) with VIQ-regressed-on-PIQ scores. Maps of correlation of VIQ-regressed-on-PIQ scores with CT, corrected for multiple comparisons using FDR. At each point on the cerebral surface, the statistical significance (probability values) of the correlation of the VIQ-regressed-on-PIQ score with CT is color coded. Warm colors (yellow, orange, red) represent a positive correlation (thicker cortex as the residual score increases), and cooler colors (blue and purple) represent a negative correlation (thinner cortex as the residual score increases). The color bar indicates the color coding of $p$ values for testing of statistical significance at each point on the surface of the brain. Top, Maps of sample 1, N=83. Bottom, Maps of sample 2, N = 58. CG, Cingulate gyrus; CUN, cuneus; FG, fusiform gyrus; GR, gyrus rectus; IFG, inferior frontal gyrus; IOG, inferior occipital gyrus; IP, inferior parietal lobule; ITG, inferior temporal gyrus, $\mathrm{LG}$, lingual gyrus; $\mathrm{MFG}$, middle frontal gyrus; $\mathrm{MOG}$, middle occipital gyrus; $\mathrm{MTG}$, middle temporal gyrus; $\mathrm{OFG}$, orbital frontal gyrus; $\mathrm{PG}$, precentral gyrus; PreCu, precuneus; $\mathrm{SFG}$, superior frontal gyrus; SMG, supramarginal gyrus; SOG, superior occipital gyrus; SPG, superior parietal gyrus; STG, superior temporal gyrus.

ence at each voxel, as well as the PIQ-regressed-on-VIQ score and the left-right CT difference at each voxel.

\section{Statistical analysis}

We used multivariate linear regression at each point on the reference surface to examine associations of the VIQ-regressed-on-PIQ and PIQregressed-on-VIQ scores with CT. In addition to the main effects, we considered for inclusion in the model the two-way interactions of age (in years) and sex (male, female) with VIQ-regressed-on-PIQ and PIQregressed-on-VIQ scores by assessing their significance in the following linear regressions:

$$
\begin{array}{r}
\mathrm{CT} i=\beta 0+\beta 1 \times \text { age }+\beta 2 \times \operatorname{sex}+\beta 3 \times \mathrm{VIQ}-\text { regressed } \\
- \text { on }- \text { PIQ score }+\beta 4 \times \mathrm{VIQ}-\text { regressed }- \text { on }- \text { PIQ score } \\
\times \operatorname{sex}(1
\end{array}
$$

$\mathrm{CT} i=\beta 0+\beta 1 \times$ age $+\beta 2 \times$ sex $+\beta 3 \times \mathrm{PIQ}-$ regressed

$$
\text { - on - VIQ score }+\beta 4 \times \text { PIQ }- \text { regressed }- \text { on }- \text { VIQ score }
$$

$$
\begin{array}{r}
\mathrm{CT} i=\beta 0+\beta 1 \times \text { age }+\beta 2 \times \text { sex }+\beta 3 \times \mathrm{VIQ}-\text { regressed } \\
- \text { on }- \text { PIQ score }+\beta 4 \times \mathrm{VIQ}-\text { regressed }- \text { on }- \text { PIQ score } \\
\times \text { age } \\
\begin{array}{r}
\mathrm{CT} i=\beta 0+\beta 1 \times \text { age }+\beta 2 \times \text { sex }+\beta 3 \times \mathrm{PIQ}-\text { regressed } \\
- \text { on }- \text { VIQ score }+\beta 4 \times \mathrm{PIQ}-\text { regressed }- \text { on }- \text { VIQ score } \\
\times \text { age },(4)
\end{array}
\end{array}
$$

where CTi is the set of CT measurements for all $i$ participants.

The models were hierarchically well formulated (all lower-order component terms were included in the model, regardless of statistical significance). We used the false detection rate (FDR; Benjamini and Hochberg, 1995) to account for the multiple correlations computed across the cortical surface. We computed the correlation of $\mathrm{CT}_{i}$ with VIQ-regressedon-PIQ score, and $\mathrm{CT}_{i}$ with PIQ-regressed-on-VIQ score. We evaluated the $p$ values of these correlations using Student's $t$ tests. Statistical maps were generated to display color-coded $p$ values across the entire surface of the cortex. Statistical maps were also generated for the interactions of sex 


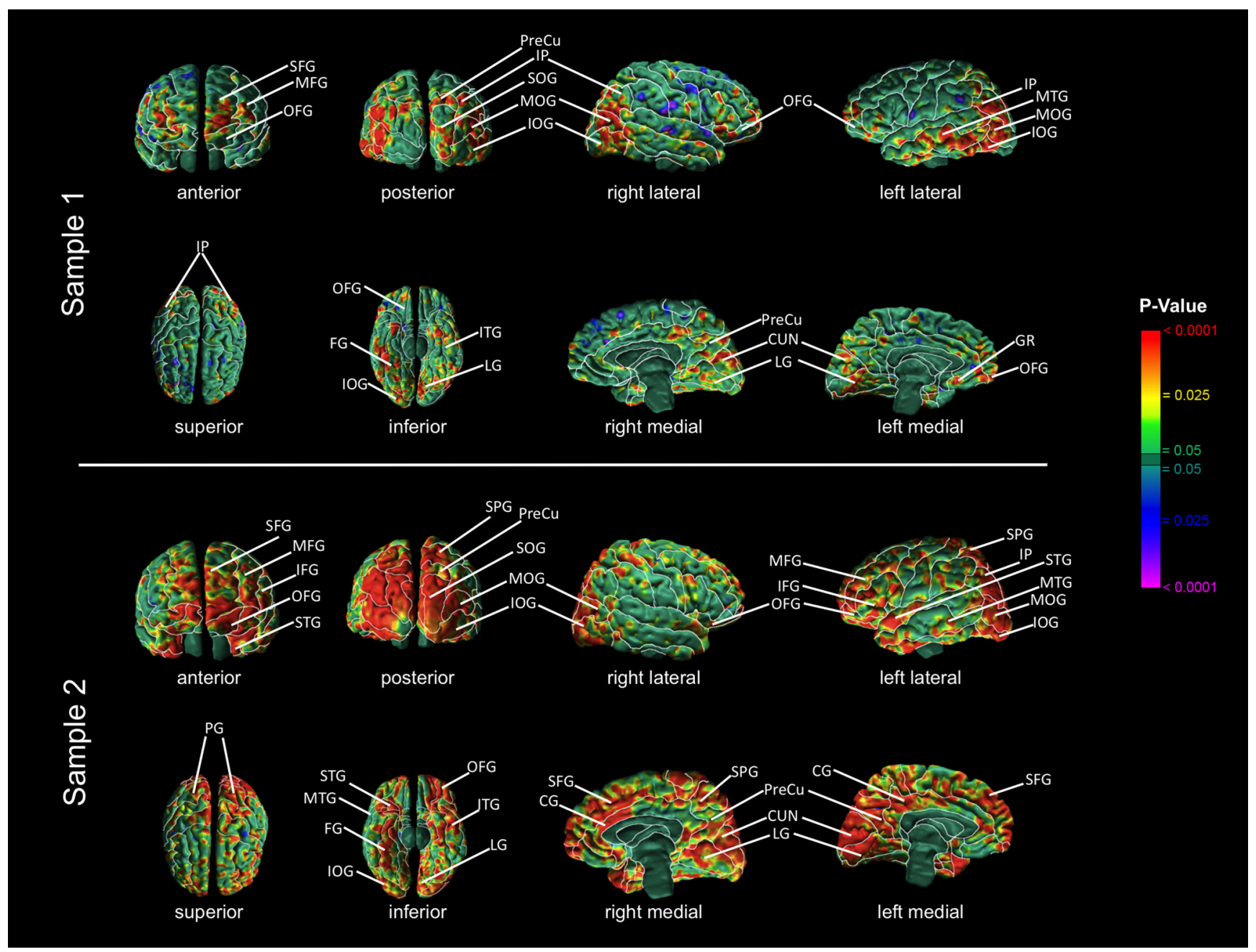

Figure 2. Significance maps showing correlations of cortical thickness (CT) with PIQ-regressed-on-VIQ scores. Maps of correlation of PIQ-regressed-on-VIQ scores with CT, corrected for multiple comparisons using FDR. At each point on the cerebral surface, the statistical significance (probability values) of the correlation of the PIQ-regressed-on-VIQ score with CT is color coded. Warm colors (yellow, orange, red) represent a positive correlation (thicker cortex as the residual score increases), and cooler colors (blue and purple) represent a negative correlation (thinner cortex as the residual score increases). The color bar indicates the color coding of $p$ values for the testing of statistical significance at each point on the surface of the brain. Top, Maps of sample 1, $N=83$. Bottom, Maps of sample 2, N = 58. CG, Cingulate gyrus; CUN, cuneus; FG, fusiform gyrus; GR, gyrus rectus; IFG, inferior frontal gyrus; IOG, inferior occipital gyrus; IP, inferior parietal lobule; ITG, inferior temporal gyrus, LG, lingual gyrus; $\mathrm{MFG}$, middle frontal gyrus; $\mathrm{MOG}$, middle occipital gyrus; $\mathrm{MTG}$, middle temporal gyrus; $\mathrm{OFG}$, orbital frontal gyrus; $\mathrm{PG}$, precentral gyrus; PreCu, precuneus; $\mathrm{SFG}$, superior frontal gyrus; SMG, supramarginal gyrus; SOG, superior occipital gyrus; SPG, superior parietal gyrus; STG, superior temporal gyrus.

with VIQ-regressed-on-PIQ score, age with VIQ-regressed-on-PIQ score, sex with PIQ-regressed-on-VIQ score, and age with PIQregressed-on-VIQ score. As none of these interactions were statistically significant, they were removed from the final statistical model.

To assess hemispheric asymmetry, the differences between left and right CTs were regressed first on age (in years), sex (male, female), and VIQ-regressed-on-PIQ residual score, and then, in a separate analysis, the differences between left and right CTs were regressed on age, sex, and PIQ-regressed-on-VIQ residual score. The statistically significant results were color encoded and displayed on the surface of the template left hemisphere, with warm colors (red and yellow) representing positive correlations (increased thickness) and cold colors (blue and purple) representing inverse correlations (reduced thickness) of thickness differences with VIQ-regressed-on-PIQ and PIQ-regressed-on-VIQ residual scores.

\section{Results}

We imaged 141 individuals, 83 (50 children, 33 adults) in sample 1 and 58 (26 children, 32 adults) in the sample 2. The mean FSIQ scores in the two samples were somewhat higher than the mean in the standardization sample (113 in sample $1 ; 121.7$ in sample 2;
100 in standardization sample), but the ranges of the VIQregressed-on-PIQ and the PIQ-regressed-on-VIQ residual scores were normally distributed around a mean of zero in each sample. The VIQ-regressed-on-PIQ score and the PIQ-regressed-onVIQ score correlated only weakly with FSIQ $\left(r^{2}=0.305\right.$ and -0.291 , respectively; $p$ values $<0.005$ ). The proportion of participants who had at least $1 \mathrm{SD}$ of difference (15 points) between VIQ and PIQ (34\% in sample 1; 40\% in sample 2) was similar to rates in normative samples (e.g., $26 \%$ in the WAIS-IV standardization sample; Wechsler, 2008). Similar to normative samples, approximately half of the participants who had at least $1 \mathrm{SD}$ of difference between VIQ and PIQ had a VIQ greater than PIQ (57\% in sample $1 ; 48 \%$ in sample 2 ), and half had a PIQ greater than VIQ (43\% in sample 1; 52\% in sample 2).

In both samples, CT correlated inversely with the magnitude of the VIQ-regressed-on-PIQ residual score and positively with the PIQ-regressed-on-VIQ residual score in both the right and left hemispheres within the anterior-most surface of the frontal lobe and within the occipital lobes (Figs. 1, 2). Specifically, ante- 

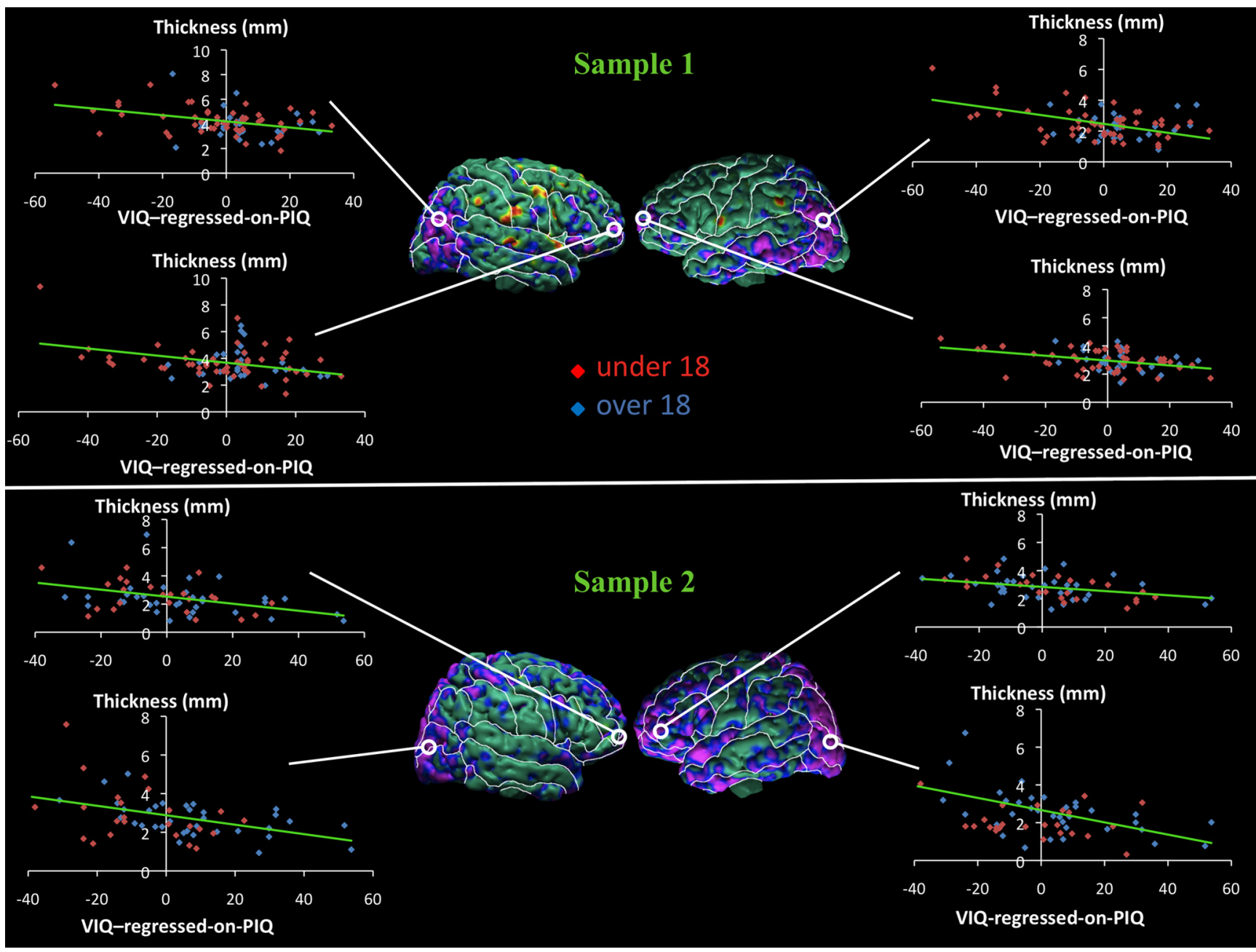

Figure 3. Scatterplots for correlations of cortical thickness (CT) with VIQ-regressed-on-PIQ scores. Maps of correlation of VIQ-regressed-on-PIQ scores with CT, corrected for multiple comparisons using FDR, and scatterplots. In the scatterplots, CTs (in millimeters) are plotted on the $y$-axis, and the VIQ-regressed-on-PIQ scores are plotted on the $x$-axis. The scatterplots show the inverse relationship between CT and VIQ-regressed-on-PIQ scores in the right and left anterior and posterior regions of the cortex in both samples. CT declines as the VIQ-regressed-on-PIQ score becomes more positive in all regions. For CT with VIQ-regressed-on-PIQ score in right posterior cortex, $r=-0.38(p<0.0004)$ in sample 1 , and $r=-0.46(p<0.0003)$ in sample 2; in left anterior cortex, $r=-0.38(p<0.0004)$ in sample 1 , and $r=-0.47(p<0.0001)$ in sample 2. Top, Maps of sample $1, N=83$. Bottom, Maps of sample $2, N=58$.

rior regions included the inferior frontal (sample 2), middle frontal (both samples), superior frontal (both samples), orbitofrontal (both samples), and superior temporal (sample 2) gyri. Posterior regions included the inferior occipital gyrus (both samples), middle occipital gyrus (both samples), superior occipital gyrus (both samples), inferior parietal lobule (both samples), parietal gyrus (sample 2), superior parietal gyrus (sample 2), middle temporal gyrus (both samples), cuneus (both samples), precuneus (both samples), and supramarginal gyrus (sample 2). Additional correlations detected on the inferior surface of the brain included the inferior temporal (both samples), fusiform (both samples), lingual (both samples), and inferior orbitofrontal gyri (both samples). Additional regions detected on the mesial surface of the brain included the gyrus rectus (sample 1) and cingulate gyrus (sample 2).

The anterior regions where CT correlated with the VIQ-PIQ discrepancy encompassed Brodmann's areas (BAs) 10, 11, and 47 in samples 1 and 2, and, additionally, include BAs 44, 45, and 46 in sample 2. BA 10 coordinates activity across multiple cognitive systems (Ramnani and Owen, 2004); BAs 11, 46, and 47 generally support behavioral inhibition (Schilling et al., 2012); and BAs 44 and 45 (Broca's region) support expressive language functions
(Foundas et al., 1996). The posterior regions where CT correlated with the VIQ-PIQ discrepancy encompassed BAs 18/19, 37, and $39 / 22$ in sample 1 ; and BAs 17, 18, 19, 7, 20, 38, and 22 in sample 2. BAs $17,18,19,20,37$, and 38 support visual processing of images and object recognition (Muller-Forell, 2005), and word recognition (Cohen et al., 2004); BA 7 supports the integration of multisensory data (Muller-Forell, 2005); and BAs 39 and 22 (Wernicke's region) support receptive language (Binder et al., 1997). On the mesial surface, regions where CT correlated with the VIQ-PIQ discrepancy encompassed BAs 23, 24, and 32 in sample 2. These areas process feedback, detect changes in the environment, and allow for alterations of behavior in response to these signals (Pearson et al., 2011).

Scatterplots showing the association of CT with the VIQregressed-on-PIQ score within these regions of statistical significance show consistently in each sample that with progressive thinning of the cortical mantle, the VIQ-regressed-on-PIQ score becomes more positive (i.e., VIQ > PIQ). In addition, as the cortical mantle thickens, the VIQ-regressed-on-PIQ score becomes increasingly more negative (i.e., PIQ > VIQ; Fig. 3). Similarly, with progressive thickening of the cortical mantle, the PIQ-regressed-onVIQ score becomes more positive (i.e., PIQ > VIQ), and as the 

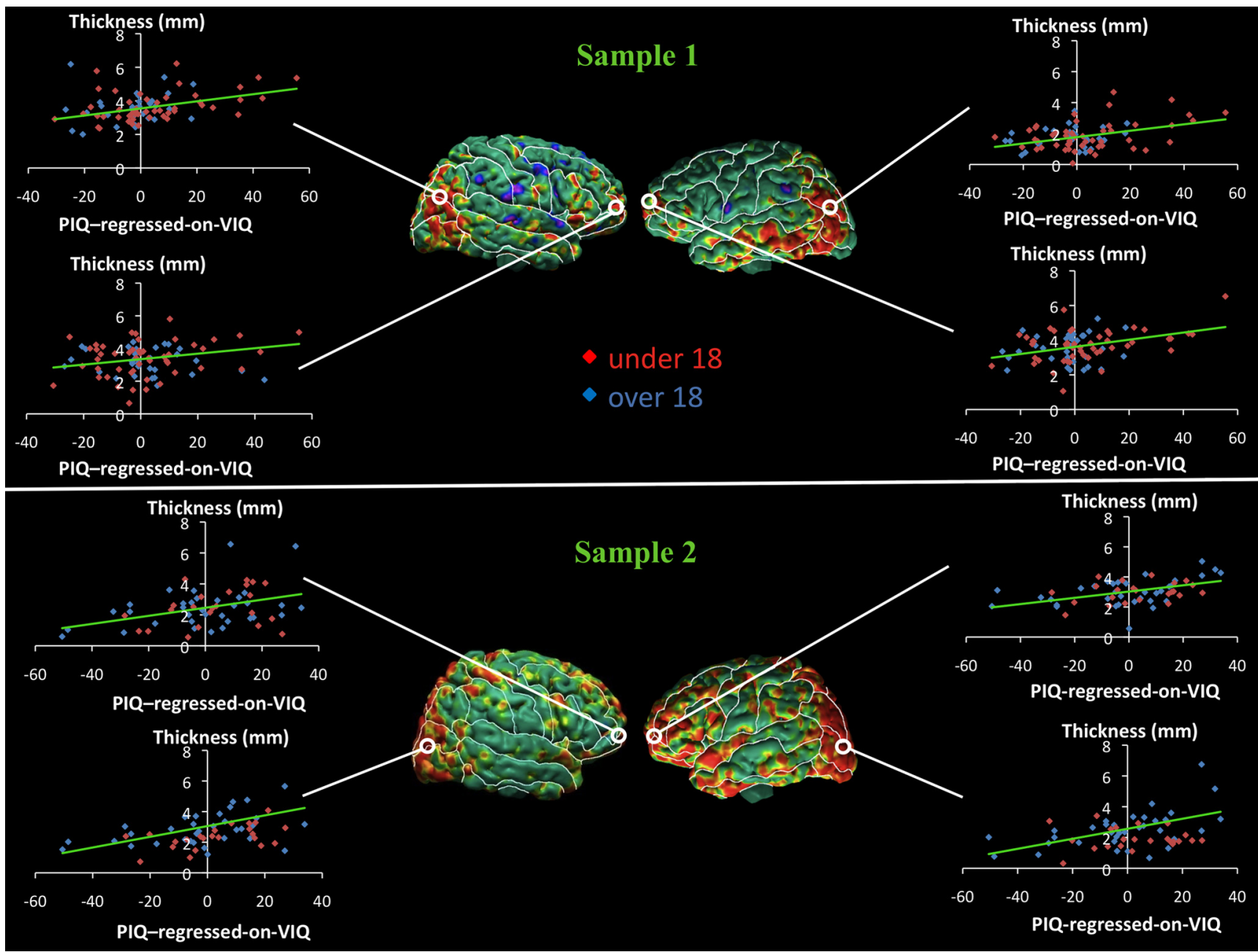

Thickness (mm)
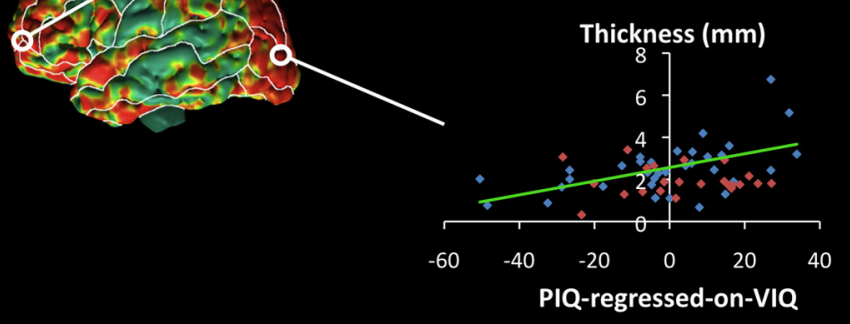

Figure 4. Scatterplots for correlations of cortical thickness (CT) with PIQ-regressed-on-VIQ scores. Maps of correlation of PIQ-regressed-on-VIQ with CT, corrected for multiple comparisons using FDR, and scatterplots. In the scatterplots, (Ts (in millimeters) are plotted on the $y$-axis, and the PIQ-regressed-on-VIQ scores are plotted on the $x$-axis. The scatterplots show the positive relationship between CT and PIQ-regressed-on-VIQ scores in the right and left anterior and posterior regions of the cortex in both samples. (T increases as the PIQ-regressed-on-VIQ score becomes more positive in all regions. For CT with PIQ-regressed-on-VIQ in right posterior cortex, $r=0.35(p<0.0012)$ in sample 1 , and $r=0.48(p<0.0002)$ in sample 2; in left anterior cortex, $r=0.34(p<0.0015)$ in sample 1, and $r=0.42(p<0.0009)$ in sample 2. Top, Maps of sample 1, $N=83$. Bottom, Maps of sample $2, N=58$.

cortical mantle thins, the PIQ-regressed-on-VIQ score becomes increasingly more negative (i.e., VIQ > PIQ; Fig. 4). Neither the VIQregressed-on-PIQ nor the PIQ-regressed-on-VIQ score interacted with age or sex in regions identified in the main effect in the regression model in either sample.

We compared the regions identified as correlating with VIQPIQ discrepancy in both the $1.5 \mathrm{~T}$ and $3 \mathrm{~T}$ samples with the regions previously identified as correlating with general intelligence. First, we compared our findings to the regions identified in a metareview of 37 studies of the neural correlates of general intelligence (Jung and Haier, 2007). The two networks have the following areas in common: BAs 10, 47, 18, and 19. Regions we identified as correlating with the VIQ-PIQ discrepancy but not with general intelligence are BAs 11 and 22. Regions that correlate with general intelligence but not with VIQ-PIQ discrepancy are BAs 6, 9, 32, 45, 46, 40, and 21. Second, we compared our findings to the regions identified as neural correlates of FSIQ within the same 1.5 T sample as in our present study (Goh et al., 2011) and identified only BAs 10 and 11.

The VIQ-PIQ discrepancy has long been considered a putative behavioral marker of psychiatric disorders, including speechlanguage impairment (Rice et al., 2005), autism spectrum disor- ders (Mayes, 2003; Charman, 2011), and nonverbal learning disability (Grodzinsky et al., 2010). Clinicians rely on the VIQPIQ difference score, not residual scores, to estimate the discrepancy between abilities. We therefore compared the regions identified as correlating with VIQ-regressed-on-PIQ and PIQregressed-on-VIQ residual scores with the regions identified as correlating with the VIQ-PIQ difference score (Fig. 5). This comparison demonstrated similar results for residual and difference scores within the anterior-most surface of the frontal lobe and within the occipital lobes, although the residual scores demonstrated stronger effects. The similarity across the type of measures indicates the validity of both residual scores and difference scores.

We also assessed whether the VIQ-regressed-on-PIQ and PIQ-regressed-on-VIQ scores correlate with measures of anatomical asymmetry in CT. To measure asymmetry in CT at each point along the cerebral surface of each participant, we established correspondences across all points on the surface of the right hemisphere with those on the surface of the left hemisphere and mapped these to the surface of the template brain, thereby allowing us to calculate asymmetry at each point as the CT in the left hemisphere minus the CT in the right hemisphere. We detected a significant inverse relationship between the magnitude of 


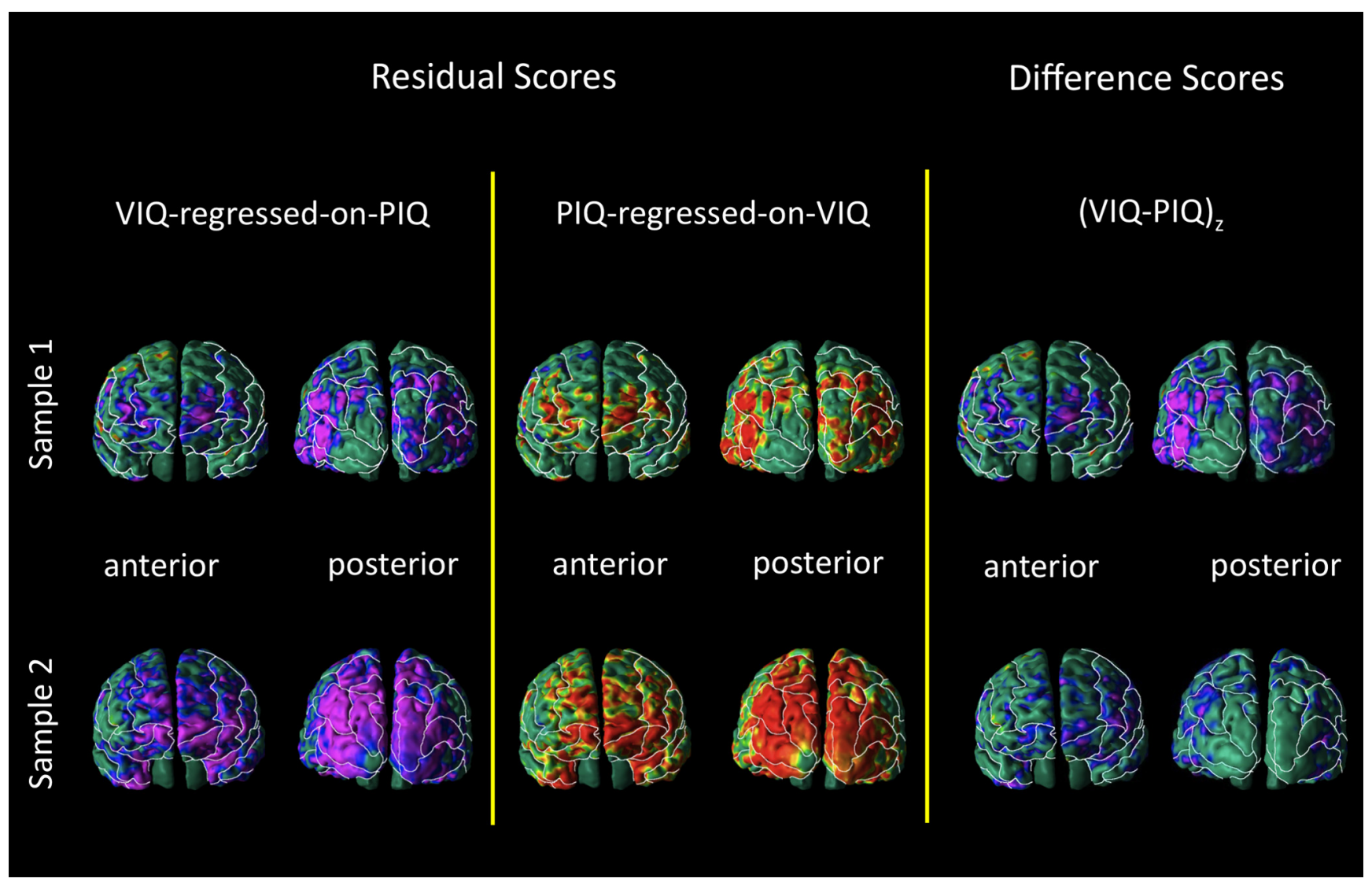

Figure 5. Significance maps showing correlations of cortical thickness (CT) with VIQ-regressed-on-PIQ score, CT with PIQ-regressed-on-VIQ score, and CT with VIQ-PIQ difference score. Anterior

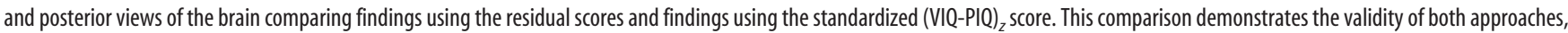
as they show similar results. Findings for the difference score analysis are included because the difference score is widely used in clinical practice. Left, Maps showing correlations of CT with VIQ-regressed-on-PIQ score. Middle, Maps showing correlations of (T with PIQ-regressed-on-VIQ score. Right, Maps showing correlations of CT with (VIQ-PIQ) z difference score. Top, Maps of sample $1, N=83$. Bottom, Maps of sample $2, N=58$.

the VIQ-regressed-on-PIQ score and the degree of CT asymmetry in inferior occipital (sample 1), middle occipital (sample 1), middle temporal (sample 1), superior temporal (both samples), precentral (sample 1), inferior frontal (both samples), and middle frontal (both samples) gyri (Fig. 6). We detected a significant positive relationship between the magnitude of the PIQregressed-on-VIQ score and the degree of CT asymmetry in the same regions listed above. Statistical significance in sample 2 is smaller than in sample 1, but the sample contained fewer subjects and the images were obtained on scanners of differing strength; overall, the direction of effect is similar in both samples, albeit sample 1 shows greater effects than sample 2. Scatterplots in all significant regions in both samples show that as VIQ-regressedon-PIQ score increases (VIQ > PIQ), left-right hemisphere CT decreases. As PIQ-regressed-on-VIQ score increases, left-right hemisphere CT increases. Thus, thinner left hemisphere CT relative to right hemisphere CT was associated with higher verbal score relative to performance score (VIQ $>$ PIQ), and thicker left hemisphere CT relative to right hemisphere CT was associated with higher performance relative to verbal score (PIQ > VIQ; Fig. 6).

\section{Discussion}

We have identified regional components of a putative neural system that underlies one well known but little-studied source of variance within and between individuals in domain-specific abilities, the VIQ-PIQ discrepancy. We detected significant thinning of anterior and posterior cortices associated with an increasing difference between verbal and performance IQ scores. We replicated these findings in two independent samples of healthy individuals, from youth to adulthood, on different age-appropriate versions of IQ tests and on two scanners of differing field strengths.

The brain regions we identified as underlying the VIQ-PIQ discrepancy support specific aspects of information processing relevant to performance on VIQ and PIQ tasks. In both samples, anterior regions encompassed BA 10, which coordinates activity across multiple cognitive systems (Ramnani and Owen, 2004), and BAs 11/47, supporting behavioral inhibition (Schilling et al., 2012). Posterior regions encompassed BAs 18/19, supporting object (Muller-Forell, 2005) and word recognition (Cohen and Dehaene, 2004), and BA 22, supporting receptive language (Binder et al., 1997). The regions where CT correlated with VIQ-PIQ discrepancy are generally distinct from other regions that correlate with general intelligence (Jung and Haier, 2007; Goh et al., 2011). Thus, the results support our hypothesis that a single neural system mediates the VIQ-PIQ discrepancy.

The entire range of values (for VIQ-regressed-on-PIQ and PIQregressed-on-VIQ scores) contributed to the correlations with CT. For VIQ-regressed-on-PIQ scores, positively valued differences (VIQ > PIQ) were associated with relative cortical thinning, and negatively valued differences (VIQ $<$ PIQ) were associated with relative cortical thickening in the same regions. We detected effects in the opposite direction for PIQ-regressed-on-VIQ scores. We note 

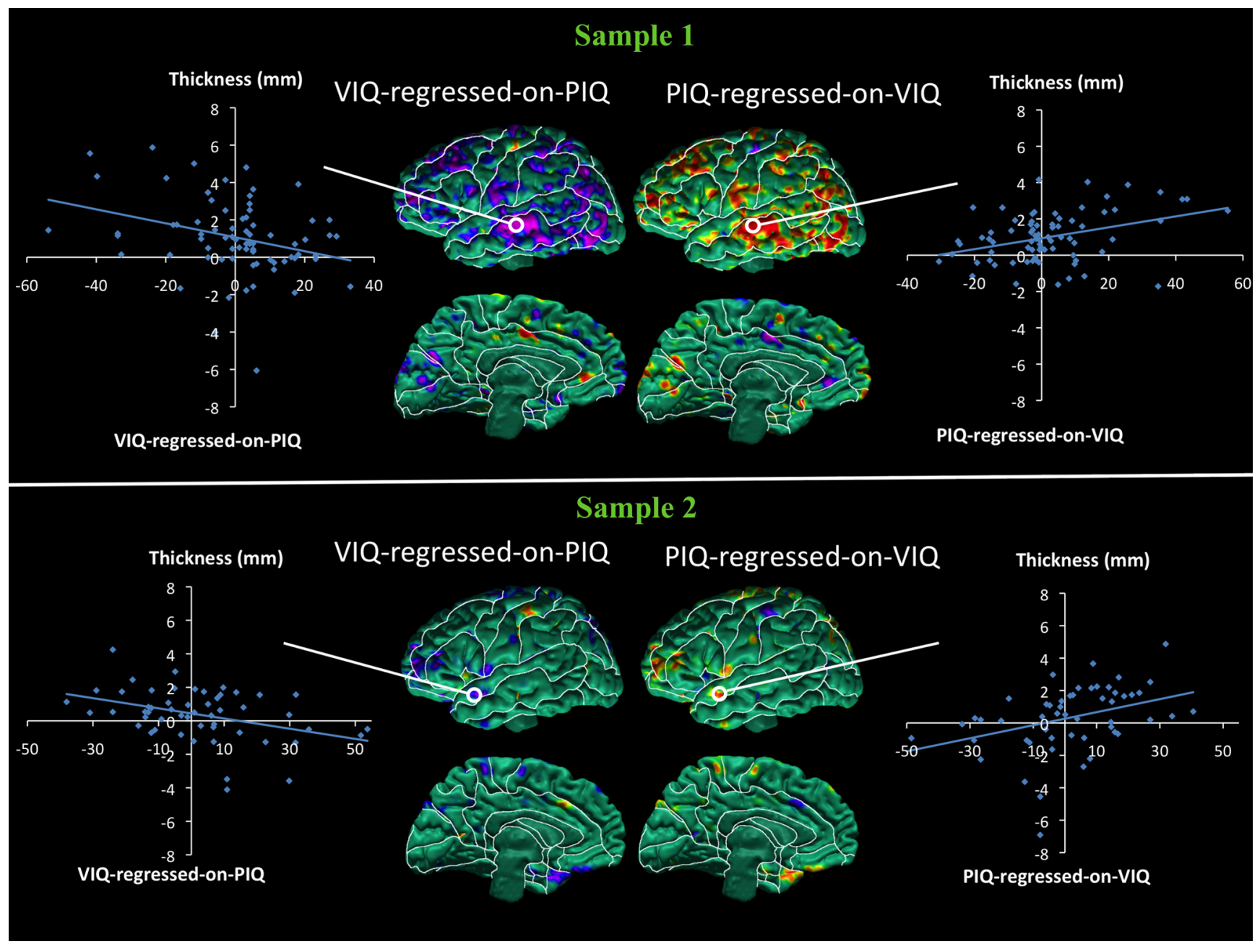

Figure 6. Correlations and scatterplots of VIQ-regressed-on-PIQ and PIQ-regressed-on-VIQ scores with asymmetry in cortical thickness (CT). Maps of correlation of VIQ-regressed-on-PIQ and PIQ-regressed-on-VIQ scores with asymmetry in CT (left hemisphere (T minus right hemisphere $\mathrm{CT}$ ), corrected for multiple comparisons using FDR. Asymmetry correlated inversely with the magnitude of the VIQ-regressed-on-PIQ score in inferior occipital (sample 1), middle occipital (sample 1), middle temporal (sample 1), superior temporal (both samples), precentral (sample 1), inferior frontal (both samples) and middle frontal (both samples) gyri (Fig. 6). We detected a significant positive relationship between the magnitude of the PIQ-regressed-on-VIQ score and the degree of CT asymmetry in the same regions listed above. Top, Maps of sample 1, $N=83$. Bottom, Maps of sample 2, $N=58$.

the opposing effects at each voxel in which thinning of the cortical mantle is associated with better performance on VIQ relative to PIQ tasks, and thickening of the cortical mantle at the same location is associated with better performance on PIQ relative to VIQ tasks.

The neural system we identified seems to operate like a CT rheostat that either improves or degrades performance of one domainspecific ability relative to the other as CT deviates either upwards or downwards from the population mean: when the cortical mantle is thin in an individual relative to the average CT of the population sample, VIQ is higher than PIQ; when the cortical mantle is thick, PIQ is higher than VIQ. In this model, the DSAs (measured with VIQ and PIQ) derive from two independent and distributed neural systems that compete, at least in part, for access to the limited capacity system, so that what access one system gains, the other loses, in a zero-sum game. The DSAs presumably rely on other circuits and brain regions in addition to the system that we have identified, but our system is presumably where the circuits that support these independent abilities physically interface with one another.

Alternatively, the opposing effects on performance of thinning and thickening at each voxel could arise if two relatively independent morphological features are present within the brain tissue imaged within each voxel, and if those two features relate in opposing ways to abilities that affect VIQ and PIQ scores. A smaller size of one feature (e.g., the thickness of one cortical layer or size of one cell type) would support better performance on VIQ tasks, and a larger size of another feature (a different cortical layer or a second cell type) would support better performance on PIQ tasks. Because CT at each voxel is a summation of the sizes of all the morphological elements within that voxel, these two morphological features and their differential associations with VIQ and PIQ tasks also would sum within each voxel, giving rise to the following four possible combinations of features that relate to the VIQ-PIQ discrepancy: (1) both morphological features are small (and thus one is contributing to higher VIQ scores and the other to lower PIQ scores): the cortical mantle is thin and VIQ is high relative to PIQ; (2) both features are large: the cortical mantle is thick, and PIQ is high relative to VIQ; (3) VIQ-related features are large and PIQ-related features are small: VIQ equals PIQ and both are low, and CT has an intermediate value nearer the population average; and (4) VIQ-related features are small and PIQrelated features are large: VIQ equals PIQ and both are high, and CT has an intermediate value near the population average. These combinations of morphological features and their relation to the VIQPIQ discrepancy can account for the linear relationship between the residual scores and CT that we detected. Therefore, the VIQ-PIQ 
discrepancy could derive from differences in morphology in two previously unidentified, independent systems supporting DSAs.

Most neuroimaging studies measure intelligence with the Wechsler scales and interpret findings in terms of verbal and performance scales. To the extent that verbal and performance scores share common requirements, they both measure general ability. In addition to this shared variance, however, each score represents unique variance associated with a domain-specific ability and variance in task-specific skills (Colom and Thompson, 2011). The constructs that these sources of variance represent have long been debated. Some IQ theorists argue that VIQ measures achievement, not ability, because performance on many of the IQ test items, such as vocabulary, depends on acquired knowledge (Naglieri and Bornstein, 2003). These theorists identify PIQ as simultaneous processing, the ability to understand the inter-relationships of variables as assessed, for example, with matrix tests (Kaufman and Kaufman, 1985; Naglieri and Das, 1997). Therefore, the VIQ-PIQ discrepancy can be interpreted as a difference between achievement and simultaneous processing. Achievement presumably represents experience and learning, whereas simultaneous processing involves a more innate maturational ability. Our findings are consistent with the known differences in the neurobiological bases for these two cognitive processes. The association of cortical thinning with higher achievement relative to simultaneous processing scores suggests that achievement may benefit from consolidative processes like synaptic or dendritic pruning. The association of cortical thickening with higher simultaneous processing relative to achievement scores suggests that simultaneous processing may benefit from greater plasticity within the cortical mantle, which presumably requires greater dendritic and axonal arborization, and greater connectivity within and across cortical layers.

Lesion studies have demonstrated that unilateral damage to a hemisphere, depending on the location of the lesion, produces loss of a skill and a discrepancy between verbal and spatial ability (Warrington et al., 1986; Gläscher et al., 2009), which at first consideration could imply a discrepancy with our findings of bilateral representation in the neural correlates of VIQ-PIQ discrepancy. Although lesion studies identify regions that are necessary for performing a particular cognitive skill, being necessary for a skill is not synonymous with being sufficient for it, as other regions may nevertheless contribute to performance measures. Human imaging studies are more likely than lesion studies to identify regions that support skills, even if not absolutely necessary for their performance. Moreover, persons with unilateral lesions often recover function through neuroplastic responses in the opposite hemisphere, suggesting the importance of bilateral contributions to verbal and spatial skills (Vigneau et al., 2011). Additionally, various stimulation studies suggest that verbal and spatial skills are represented bilaterally in the brain (Pascual-Leone et al., 1994; Binder et al., 1996; Knecht et al., 2002). Finally, differences in conclusions from morphometric and lesion studies likely relate to fundamental differences in the measures and methods used to localize the effects of interest. CT, for instance, is a feature only of gray matter, which supports primarily local processing, whereas naturalistic lesions often affect more prominently white matter, which supports communication across brain regions.

In addition to bilateral correlations, we detected evidence in both samples for a less robust correlation of hemispheric asymmetry in CT with VIQ-PIQ discrepancy. A thinner left cortex relative to right was associated with higher VIQ relative to PIQ, and a thicker left cortex relative to right was associated with higher PIQ relative to VIQ. These findings are generally consistent with the hemisphere-specific findings from naturalistic hu- man lesion studies (Gläscher et al., 2009), and they are consistent with our primary finding that higher VIQ relative to PIQ is associated with cortical thinning and higher PIQ relative to VIQ is associated with cortical thickening.

Studies of patient populations suggest that the VIQ-PIQ discrepancy may be under genetic control. The VIQ-PIQ discrepancy in autism defines a particular phenotype that is linked to two genes, one of which is also implicated in speech-language impairment (Chapman et al., 2011). The presence of the VIQ-PIQ discrepancy in Prader-Willi syndrome depends on the disease genotype (either a deletion or uniparental disomy) and is associated with higher risk for associated autism spectrum behaviors (Copet et al., 2010).

Our study has limitations. To evaluate whether our results vary as a function of overall IQ would require a sufficiently large sample to assess these correlates separately in groups of people with low, medium, or high FSIQ. To assess the histological correlates of our findings would require a large postmortem study. Our participant samples spanned a wide range of ages, which potentially could add variability to the correlations that we assessed. Age did not interact with VIQ-PIQ discrepancy, however, suggesting that the association of VIQ-PIQ discrepancy with CT is consistent across ages. The inclusion of participants across age and developmental stages can be viewed as a relative strength of our study, as it increases the generalizability of our findings. The contributions of even the youngest children in our samples to the correlations of CT with VIQ-PIQ discrepancy (Figs. 3, 4) suggest that the morphological and cellular determinants of the discrepancies in these cognitive domains may be present early in life.

\section{References}

Anastasi A, Urbina S (1997) Psychological testing. Upper Saddle River, NJ: Prentice Hall.

Andreasen NC, Flaum M, Swayze V 2nd, O'Leary DS, Alliger R, Cohen G, Ehrhardt J, Yuh WT (1993) Intelligence and brain structure in normal individuals. Am J Psychiatry 150:130-134. Medline

Arndt S, Cohen G, Alliger RJ, Swayze VW 2nd, Andreasen NC (1991) Problems with ratio and proportion measures of imaged cerebral structures. Psychiatry Res 40:79-89. Medline

Bansal R, Staib LH, Whiteman R, Wang YM, Peterson BS (2005) ROCbased assessments of $3 \mathrm{D}$ cortical surface-matching algorithms. Neuroimage 24:150-162. CrossRef Medline

Benjamini Y, Hochberg Y (1995) Controlling the false discovery rate: a practical and powerful approach to multiple testing. J R Stat Soc B 57: 289-300.

Binder JR, Swanson SJ, Hammeke TA, Morris GL, Mueller WM, Fischer M Benbadis S, Frost JA, Rao SM, Haughton VM (1996) Determination of language dominance using functional MRI: a comparison with the Wada test. Neurology 46:978-984. CrossRef Medline

Binder JR, Frost JA, Hammeke TA, Cox RW, Rao SM, Prieto T (1997) Human brain language areas identified by functional magnetic resonance imaging. J Neurosci 17:353-362. Medline

Canivez GL, Neitzel R, Martin BE (2005) Construct validity of the Kaufman Brief Intelligence Test, Wechsler Intelligence Scale for Children-third edition, and Adjustment Scales for Children and Adolescents. J Psychoeduc Assess 23:15-34. CrossRef

Carroll JB (1993) Human cognitive abilities: a survey of factor-analytic studies. Cambridge, UK: Cambridge UP.

Chapman NH, Estes A, Munson J, Bernier R, Webb SJ, Rothstein JH, Minshew NJ, Dawson G, Schellenberg GD, Wijsman EM (2011) Genomescan for IQ discrepancy in autism: evidence for loci on chromosomes 10 and 16. Hum Genet 129:59-70. CrossRef Medline

Charman T, Pickles A, Simonoff E, Chandler S, Loucas T, Baird G (2011) IQ in children with autism spectrum disorders: data from the Special Needs and Autism Project (SNAP). Psychol Med 41:619-627. CrossRef Medline

Cohen L, Dehaene S (2004) Specialization within the ventral stream: the case for the visual word form area. Neuroimage 22:466-476. CrossRef Medline 
Cohen L, Jobert A, Le Bihan D, Dehaene S (2004) Distinct unimodal and multimodal regions for word processing in the left temporal cortex. Neuroimage 23:1256-1270. CrossRef Medline

Colom R, Thompson PM (2011) Understanding human intelligence by imaging the brain. In: The Wiley-Blackwell handbook of individual differences (Chamorro-Premuzic T, von Stumm S, Furnham A, eds), pp 330-352. Hoboken, NJ: Wiley-Blackwell.

Copet P, Jauregi J, Laurier V, Ehlinger V, Arnaud C, Cobo AM, Molinas C, Tauber M, Thuilleaux D (2010) Cognitive profile in a large French cohort of adults with Prader-Willi syndrome: differences between genotypes. J Intellect Disabil Res 54:204-215. CrossRef Medline

Flanagan D, McGrew KS, Ortiz SO (2000) The Wechsler Intelligence Scales and Gf-Gc theory. Boston: Allyn \& Bacon.

Foundas AL, Leonard CM, Gilmore RL, Fennell EB, Heilman KM (1996) Pars triangularis asymmetry and language dominance. Proc Natl Acad Sci U S A 93:719-722. CrossRef Medline

Gläscher J, Tranel D, Paul LK, Rudrauf D, Rorden C, Hornaday A, Grabowski T, Damasio H, Adolphs R (2009) Lesion mapping of cognitive abilities linked to intelligence. Neuron 61:681-691. CrossRef Medline

Goh S, Bansal R, Xu D, Hao X, Liu J, Peterson BS (2011) Neuroanatomical correlates of intellectual ability across the life span. Dev Cogn Neurosci 1:305-312. CrossRef Medline

Gray JR, Chabris CF, Braver TS (2003) Neural mechanisms of general fluid intelligence. Nat Neurosci 6:316-322. CrossRef Medline

Grodzinsky GM, Forbes PW, Bernstein JH (2010) A practice-based approach to group identification in nonverbal learning disorders. Child Neuropsychol 16:433-460. CrossRef Medline

Haralick RM, Shapiro LG (1991) Computer and robot vision. Boston: Addison-Wesley.

Jung RE, Haier RJ (2007) The Parieto-Frontal Integration Theory (P-FIT) of intelligence: converging neuroimaging evidence. Behav Brain Sci 30: 135-154. CrossRef Medline

Kamphaus RW (2005) Clinical assessment of child and adolescent intelligence. New York: Springer.

Karama S, Ad-Dab'bagh Y, Haier RJ, Deary IJ, Lyttelton OC, Lepage C, Evans AC (2009) Positive association between cognitive ability and cortical thickness in a representative US sample of healthy 6 to 18 year-olds. Intelligence 37:145-155. CrossRef Medline

Karama S, Colom R, Johnson W, Deary IJ, Haier R, Waber DP, Lepage C, Ganjavi H, Jung R, Evans AC (2011) Cortical thickness correlates of specific cognitive performance accounted for by the general factor of intelligence in healthy children aged 6 to 18 . Neuroimage 55:1443-1453. CrossRef Medline

Kaufman AS, Kaufman NL (1985) Kaufman Brief Intelligence Test. San Antonio, TX: Pearson.

Kaufman A, Kaufman J, Balgopaland R, McLean JE (1996) Comparison of three WISC-III short forms: weighing psychometric, clinical, and practical factors. J Clin Child Psychol 25:97-105. CrossRef

Kaufman J, Birmaher B, Brent D, Rao U, Flynn C, Moreci P, Williamson D, Ryan N (1997) Schedule for Affective Disorders and Schizophrenia for School-Age Children-Present and Lifetime Version (K-SADS-PL): initial reliability and validity data. J Am Acad Child Adolesc Psychiatry 36:980988. CrossRef Medline

Knecht S, Flöel A, Dräger B, Breitenstein C, Sommer J, Henningsen H, Ringelstein EB, Pascual-Leone A (2002) Degree of language lateralization determines susceptibility to unilateral brain lesions. Nat Neurosci 5:695699. CrossRef Medline

Lange N, Froimowitz MP, Bigler ED, Lainhart JE (2010) Associations between IQ, total and regional brain volumes, and demography in a large normative sample of healthy children and adolescents. Dev Neuropsychol 35:296-317. CrossRef Medline

Luders E, Narr KL, Bilder RM, Thompson PM, Szeszko PR, Hamilton L, Toga AW (2007) Positive correlations between corpus callosum thickness and intelligence. Neuroimage 37:1457-1464. CrossRef Medline

Marr D, Hildreth E (1980) Theory of edge detection. Proc R Soc Lond B Biol Sci 207:187-217. CrossRef Medline

Mayes SD, Calhoun SL (2003) Ability profiles in children with autism: influence of age and IQ. Autism 7:65-80. CrossRef Medline

Mazziotta J, Toga A, Evans A, Fox P, Lancaster J, Zilles K, Woods R, Paus T, Simpson G, Pike B, Holmes C, Collins L, Thompson P, MacDonald D, Iacoboni M, Schormann T, Amunts K, Palomero-Gallagher N, Gever S, Parsons L, et al. (2001) A probabilistic atlas and reference system for the human brain: International Consortium for Brain Mapping (ICBM). Philos Trans R Soc Lond B Biol Sci 356:1293-1322. CrossRef Medline

Muller-Forell WS (2005) Imaging of orbital and visual pathway pathology. New York: Springer.

Naglieri JA, Bornstein BT (2003) Intelligence and achievement: just how correlated are they? J Psychoeduc Assess 21:244-260. CrossRef

Naglieri JA, Das JP (1997) Cognitive assessment system interpretive handbook. Rolling Meadows, IL: Riverside Publishing.

Narr KL, Woods RP, Thompson PM, Szeszko P, Robinson D, Dimtcheva T, Gurbani M, Toga AW, Bilder RM (2007) Relationships between IQ and regional cortical gray matter thickness in healthy adults. Cereb Cortex 17:2163-2171. CrossRef Medline

Packard MG (2009) Anxiety, cognition, and habit: a multiple memory systems perspective. Brain Res 1293:121-128. CrossRef Medline

Pascual-Leone A, Gomez-Tortosa E, Grafman J, Alway D, Nichelli P, Hallett M (1994) Induction of visual extinction by rapid-rate transcranial magnetic stimulation of parietal lobe. Neurology 44:494-498. CrossRef Medline

Pearson JM, Heilbronner SR, Barack DL, Hayden BY, Platt ML (2011) Posterior cingulate cortex: adapting behavior to a changing world. Trends Cogn Sci 15:143-151. CrossRef Medline

Plessen KJ, Bansal R, Zhu H, Whiteman R, Amat J, Quackenbush GA, Martin L, Durkin K, Blair C, Royal J, Hugdahl K, Peterson BS (2006) Hippocampus and amygdala morphology in attention-deficit/hyperactivity disorder. Arch Gen Psychiatry 63:795-807. CrossRef Medline

Ramnani N, Owen AM (2004) Anterior prefrontal cortex: insights into function from anatomy and neuroimaging. Nat Rev Neurosci 5:184-194. CrossRef Medline

Rice ML, Warren SF, Betz SK (2005) Language symptoms of developmental language disorders: an overview of autism, Down syndrome, fragile $\mathrm{X}$, specific language impairment, and Williams syndrome. Appl Psycholinguist 26:7-27. CrossRef

Rosenfeld A, Pfaltz JL (1968) Distance functions on digital pictures. Pattern Recognit 1:33-61.

Schilling C, Kühn S, Romanowski A, Schubert F, Kathmann N, Gallinat J (2012) Cortical thickness correlates with impulsiveness in healthy adults. Neuroimage 59:824-830. CrossRef Medline

Shattuck DW, Leahy RM (2002) BrainSuite: an automated cortical surface identification tool. Med Image Anal 6:129-142. CrossRef Medline

Shaw P, Greenstein D, Lerch J, Clasen L, Lenroot R, Gogtay N, Evans A, Rapoport J, Giedd J (2006) Intellectual ability and cortical development in children and adolescents. Nature 440:676-679. CrossRef Medline

Shrout PE, Fleiss JL (1979) Intraclass correlations: uses in assessing rater reliability. Psychol Bull 86:420 - 428. CrossRef Medline

Sled JG, Zijdenbos AP, Evans AC (1998) A nonparametric method for automatic correction of intensity nonuniformity in MRI data. IEEE Trans Med Imaging 17:87-97. CrossRef Medline

Spitzer RL, Williams JB, Gibbon M, First MB (1992) The structured clinical interview for DSM-III-R (SCID). I: history, rationale, and description. Arch Gen Psychiatry 49:624-629. CrossRef Medline

Vigneau M, Beaucousin V, Hervé PY, Jobard G, Petit L, Crivello F, Mellet E, Zago L, Mazoyer B, Tzourio-Mazoyer N (2011) What is righthemisphere contribution to phonological, lexico-semantic, and sentence processing? Insights from a meta-analysis. Neuroimage 54:577-593. CrossRef Medline

Warrington EK, James M, Maciejewski C (1986) The WAIS as a lateralizing and localizing diagnostic instrument: a study of 656 patients with unilateral cerebral lesions. Neuropsychologia 24:223-239. CrossRef Medline

Watkins MW (2006) Orthogonal higher order structure of the Wechsler Intelligence Scale for Children—fourth edition. Psychol Assess 18:123125. CrossRef Medline

Wechsler D (1981) Wechsler adult intelligence scale, revised. San Antonio: The Psychological Corporation.

Wechsler D (1991) Wechsler intelligence scale for children, third edition. San Antonio: The Psychological Corporation.

Wechsler D (1999) Wechsler abbreviated scale of intelligence. San Antonio: The Psychological Corporation.

Wechsler D (2008) Wechsler adult intelligence scale, fourth edition: technical manual. San Antonio: The Pscyhological Corporation.

Witelson SF, Beresh H, Kigar DL (2006) Intelligence and brain size in 100 postmortem brains: sex, lateralization and age factors. Brain 129:386398. CrossRef Medline 